\title{
The Similar Structure Method for Solving the Model of Fractal Dual-Porosity Reservoir
}

\author{
Li Xu, ${ }^{1}$ Xiangjun Liu, ${ }^{1}$ Lixi Liang, ${ }^{1}$ Shunchu Li, ${ }^{2}$ and Longtao Zhou' \\ ${ }^{1}$ State Key Laboratory of Oil and Gas Reservoir Geology and Exploitation, Southwest Petroleum University, Chengdu, China \\ ${ }^{2}$ Institute of Applied Mathematics, Xihua University, Chengdu 610039, China \\ Correspondence should be addressed to Shunchu Li; lishunchu@163.com
}

Received 27 June 2013; Revised 4 November 2013; Accepted 16 November 2013

Academic Editor: Alex Elías-Zúñiga

Copyright (c) $2013 \mathrm{Li} \mathrm{Xu}$ et al. This is an open access article distributed under the Creative Commons Attribution License, which permits unrestricted use, distribution, and reproduction in any medium, provided the original work is properly cited.

\begin{abstract}
This paper proposes a similar structure method (SSM) to solve the boundary value problem of the extended modified Bessel equation. The method could efficiently solve a second-order linear homogeneous differential equation's boundary value problem and obtain its solutions' similar structure. A mathematics model is set up on the dual-porosity media, in which the influence of fractal dimension, spherical flow, wellbore storage, and skin factor is taken into cosideration. Researches in the model found that it was a special type of the extended modified Bessel equation in Laplace space. Then, the formation pressure and wellbore pressure under three types of outer boundaries (infinite, constant pressure, and closed) are obtained via SSM in Laplace space. Combining SSM with the Stehfest algorithm, we propose the similar structure method algorithm (SSMA) which can be used to calculate wellbore pressure and pressure derivative of reservoir seepage models clearly. Type curves of fractal dual-porosity spherical flow are plotted by SSMA. The presented algorithm promotes the development of well test analysis software.
\end{abstract}

\section{Introduction}

Petroleum engineering and percolation mechanics have scale invariances in non-Euclidean heterogeneous porous media. References $[1,2]$ built mathematical models of reservoirs and described porous media by homogeneous or quasi homogeneous distribution. The reservoirs distribution and pore structure are complexities. Reference [3] demonstrated that the fractal characteristics of porous media affected fluid flow by studying many geological properties. Reference [4] discussed the geometric properties and spatial correlation structure of fractal distributions and reviewed methods for measuring the fractal character of field data and synthesizing fields with a similar correlation structure. References $[5,6]$ proposed a seepage model of Newtonian fluid in fractal reservoir and analyzed its well test curve. References $[7,8]$ showed that fractal reservoir seepage model can be explained much more effectively than traditional one and obtained a conclusion very consistent with well test curve. References [9, 10] discussed that the fractal theory was powerful in analysis of the fluid flow properties with complex and microscopic stochastic microstructures in porous media.
Model of flow through dual-porosity formations was proposed by [11]. Barenblatt et al. considered the medium of the blocks and that of the fractures as overlapping continua over entire flow domain. Darcy's law and conservation of mass equation are written separately for the fluid in each medium. A dual-porosity media has fluid exchange between the two constitutive media. Many natural reservoirs fit with this scheme, so the fluid flow model of dual-porosity is particularly popular in mining engineering and petroleum engineering. The previous studies of fractal dual-porosity seepage flow model are mostly considered on radial flow. Reference [12] demonstrated that spherical flow analysis can model the well test interpretation and provide data insight for reservoir and production study more suitably.

Reference [13] first presented the concept of solutions' similar structure for a second-order linear homogeneous differential equation and the partial differential equation which can be transformed into ordinary differential equation system via variable substitution or integral transform. Expression of their solutions can be simplified as a unified continued fraction only with different kernel functions. 
The solutions' similar structure theory of differential equation has been widely applied in engineering problems, in particular, solving of reservoir mathematical models. References [14, 15] built mathematical models for fluid flow in porous media and got their solutions' similar structure. However, most of research got their similar solutions and kernel functions via a complicated process of solutions. So, we sum up the detailed construction steps of SSM for the extended modified Bessel equation's value problem. It is shown that the solutions of the extended modified Bessel equation can be simply constructed by the coefficients of boundary without too much calculating. A spherical flow of fractal model is designed in dual-porosity reservoir. The similar structure of formation pressure and wellbore pressure in Laplace space is conveniently calculated by SSM. In Section 3, combining SSM with Stehfest [16] numerical inversion we compile a similar structure method algorithm (SSMA). Type curves of the fractal dual-porosity spherical flow model are plotted with SSMA in real time domain. In Section 4, how the fractal dimension and wellbore storage affect formation pressure and its derivative of dualporosity reservoir are discussed on infinite boundary. In a word, the SSM is a straightforward method for solving the differential equation's boundary value problems with complex boundary condition, especially in petroleum engineering seepage models. SSMA has the significance meaning for making well test analysis.

\section{The SSM for Solving the Model of the Fractal Dual-Porosity Reservoir}

2.1. The Similar Structure Method (SSM). To solve reservoir model, we first introduce SSM for the extended modified Bessel equation's boundary value problem, which frequently occurred in reservoir engineering:

$$
\begin{gathered}
x^{2} y^{\prime \prime}+A x y^{\prime}+\left(B-C x^{q}\right) y=0 \quad(x>0), \\
{\left[E y+(1+E F) y^{\prime}\right]_{x=a}=D,} \\
{\left[G y+H y^{\prime}\right]_{x=b}=0,}
\end{gathered}
$$

where $A, B, C, D, F, G, H, a$, and $b$ are all known as real numbers and satisfy

$$
\begin{gathered}
(1-A)^{2}>4 B, \quad C>0, \quad D \neq 0, \quad G^{2}+H^{2} \neq 0, \\
0<a<b, \quad q \neq 0 .
\end{gathered}
$$

The SSM steps are presented as follows.

Step 1. Find two linearly independent solutions of the extended modified Bessel equation (1) (see Appendix A for details). They are

$$
x^{\alpha} I_{v}\left(k x^{\beta}\right), \quad x^{\alpha} K_{v}\left(k x^{\beta}\right),
$$

where $\alpha=(1-A) / 2, \beta=q / 2, k=\sqrt{2 C} / q$, and $v=$ $\sqrt{(1-A)^{2}-4 B / q}$.
Step 2. A binary function $\varphi(x, \xi)$ is defined by the two linearly independent solutions $x^{\alpha} I_{v}\left(k x^{\beta}\right), x^{\alpha} K_{v}\left(k x^{\beta}\right)$ :

$$
\begin{aligned}
\varphi(x, \xi)=\varphi_{0.0}(x, \xi)=(x \xi)^{\alpha}[ & K_{v}\left(k x^{\beta}\right) I_{v}\left(k \xi^{\beta}\right) \\
& \left.-I_{v}\left(k x^{\beta}\right) K_{v}\left(k \xi^{\beta}\right)\right] .
\end{aligned}
$$

If we define

$$
\begin{aligned}
\Psi_{m \cdot n}(x, y, t)= & K_{m}(x t) I_{n}(y t) \\
& +(-1)^{m-n+1} I_{m}(x t) K_{n}(y t),
\end{aligned}
$$

where $m, n$ are real numbers, the binary function $\varphi(x, \xi)$ can be rewritten as

$$
\varphi(x, \xi)=(x \xi)^{\alpha} \Psi_{v \cdot v}\left(x^{\beta}, \xi^{\beta}, k\right)
$$

Then, calculate the partial derivative of $\varphi(x, \xi)$ for $x, \xi$, respectively:

$$
\begin{aligned}
& \varphi_{1.0}(x, \xi)=\frac{\partial}{\partial x} \varphi_{0.0}(x, \xi) \\
& =x^{\alpha-1} \xi^{\alpha}\left[(\alpha+\beta v) \Psi_{v \cdot v}\left(x^{\beta}, \xi^{\beta}, k\right)\right. \\
& \left.-k \beta x^{\beta} \Psi_{v+1 \cdot v}\left(x^{\beta}, \xi^{\beta}, k\right)\right], \\
& \varphi_{0.1}(x, \xi)=\frac{\partial}{\partial \xi} \varphi_{0.0}(x, \xi) \\
& =x^{\alpha} \xi^{\alpha-1}\left[(\alpha+\beta v) \Psi_{v \cdot v}\left(x^{\beta}, \xi^{\beta}, k\right)\right. \\
& \left.+k \beta \xi^{\beta} \Psi_{v \cdot v+1}\left(x^{\beta}, \xi^{\beta}, k\right)\right], \\
& \varphi_{1.1}(x, \xi)=\frac{\partial}{\partial x} \varphi_{0.1}(x, \xi)=\frac{\partial}{\partial \xi} \varphi_{1.0}(x, \xi) \\
& =(x \xi)^{\alpha-1}\left[(\alpha+\beta v)^{2} \Psi_{v \cdot v}\left(x^{\beta}, \xi^{\beta}, k\right)\right. \\
& -k \beta(\alpha+\beta v) x^{\beta} \Psi_{v+1 \cdot v}\left(x^{\beta}, \xi^{\beta}, k\right) \\
& +k \beta(\alpha+\beta v) \xi^{\beta} \Psi_{v \cdot v+1}\left(x^{\beta}, \xi^{\beta}, k\right) \\
& \left.-k^{2} \beta^{2}(x \xi)^{\beta} \Psi_{v+1 \cdot v+1}\left(x^{\beta}, \xi^{\beta}, k\right)\right] .
\end{aligned}
$$


Step 3. A similar kernel function is constructed by using the coefficients of the right boundary (3) and Step 2 definition:

$$
\Phi(x)=\frac{G \varphi_{0.0}(x, b)+H \varphi_{0.1}(x, b)}{G \varphi_{1.0}(a, b)+H \varphi_{1.1}(a, b)} .
$$

One can easily get $\Phi(a)$.

Step 4. The similar structure of solutions is designed by the coefficients of the left boundary (2) and

$$
y=D \cdot \frac{1}{E+(1 /(F+\Phi(a)))} \cdot \frac{1}{F+\Phi(a)} \cdot \Phi(x) .
$$

Consequently, (15) is the similar solutions of the extended modified Bessel equation's boundary value problem (1)-(3).

\subsection{The SSM for Solving the Model of the Fractal Dual-Porosity} Reservoir. The dimensionless mathematical model of a fractal dual-porosity reservoir is presented in Appendix B. In order to solve the model, we assume that the fractal dimension of fissure and matrix block are identical; that is, $d_{f_{1}}=$ $d_{f_{2}}$. Ideally, all the fluid can be seeped into fissure media from matrix block. We give the hypothesis that fractal exponential in matrix block approximates at zero $(\theta=0)$. Considering spherical symmetric flow, we can write the model in the Laplace space as follows.

Fundamental differential equations:

$$
\begin{gathered}
\frac{d^{2} \bar{P}_{1 D}}{d r_{D}^{2}}+\frac{\beta_{0}}{r_{D}} \frac{d \bar{P}_{1 D}}{d r_{D}}+\frac{\lambda r_{D}^{\theta_{1}}}{e^{2 s}}\left(\bar{P}_{2 D}-\bar{P}_{1 D}\right)=0, \\
(1-\omega) \bar{P}_{2 D}+\lambda C_{D}\left(\bar{P}_{2 D}-\bar{P}_{1 D}\right)=0 .
\end{gathered}
$$

Initial boundary conditions:

$$
\bar{P}_{1 D}\left(r_{D}, 0\right)=\bar{P}_{2 D}\left(r_{D}, 0\right)=0 \text {. }
$$

Interboundary conditions:

$$
\left.\left[-z \bar{P}_{w D}\left(r_{D}, z\right)+\frac{d \bar{P}_{1 D}\left(r_{D}, z\right)}{d r_{D}}\right]\right|_{r_{D}=1}=-\bar{q}_{D}(z) .
$$

The three types of outer boundary conditions:

$$
\begin{gathered}
\bar{P}_{i D}(\infty, z)=0 \\
\bar{P}_{i D}\left(R_{D}, z\right)=0 \quad \text { or } \quad \frac{d \bar{P}_{i D}}{d r_{D}}\left(R_{D}, z\right)=0 \quad(i=1,2) .
\end{gathered}
$$

They denote that the outer boundaries of circular reservoir are infinite, constant pressure, and closed, respectively. All the notations are explained in the nomenclature section.

To simplify (17) yields

$$
\bar{P}_{2 D}=\frac{\lambda C_{D}}{(1-\omega) z+\lambda C_{D}} \bar{P}_{1 D}
$$

$\bar{P}_{2 D}$ can be expressed by $\bar{P}_{1 D}$ linearly. Next in the paper, we only consider $\bar{P}_{1 D}$. Taking (21) into (16) yields

$$
\frac{d^{2} \bar{P}_{1 D}}{d r_{D}^{2}}+\frac{\beta_{0}}{r_{D}} \frac{d \bar{P}_{1 D}}{d r_{D}}-z^{*} r^{\theta_{1}} \bar{P}_{1 D}=0
$$

where $z^{*}(z)=\left(z / C_{D} e^{2 s}\right)\left[\left(z \omega(1-\omega)+\lambda C_{D}\right) /\left(z(1-\omega)+\lambda C_{D}\right)\right]$.

Equation (23) is a special form of the extended modified Bessel equation, so we can use SSM to construct the solutions' similar structure of the dimensionless mathematical mode of fractal dual-porosity reservoir flow (16)-(20).

Equations (19), (20), and (23) comparing with (1)-(3) result in

$$
\begin{gathered}
y=\bar{P}_{1 D}, \quad x=r_{D}, \quad A=\beta_{0}, \quad B=0, \quad C=z^{*} r^{\theta}, \\
E=z, \quad F=0, \quad D=-\bar{q}_{D}(z), \quad a=1 .
\end{gathered}
$$

$G=1, H=0, b \rightarrow \infty$ or $G=1, H=0, b=R_{D}$ or $G=0, H=1, b=R_{D}$ representing three types of outer boundaries, respectively. So we can construct the similar structure of formation pressure in Laplace space as in the following expression:

$$
\bar{P}_{1 D}\left(r_{D}, z\right)=\bar{q}_{D}(z) \frac{1}{z+(1 / \Phi(1, z))} \frac{1}{\Phi(1, z)} \Phi\left(r_{D}, z\right) .
$$

$\Phi\left(r_{D}, z\right)$ is the similar kernel function.

For $\bar{P}_{1 D}(\infty, z)=0$, using the properties of modified Bessel functions $\lim _{x \rightarrow \infty} I_{v}(x)=\infty, \lim _{x \rightarrow \infty} K_{v}(x)=0$ with (14) obtaining the similar kernel function as follows:

$$
\begin{aligned}
\Phi\left(r_{D}, z\right)= & \left(r_{D}^{\left(1-\beta_{0}\right) / 2} K_{v}\left(\frac{2 \sqrt{z^{*}}}{\theta_{1}+2} r_{D}^{\left(\theta_{1} / 2\right)+1}\right)\right) \\
& \times\left(\sqrt{z^{*}} K_{v+1}\left(\frac{2 \sqrt{z^{*}}}{\theta_{1}+2}\right)-\left(1-\beta_{0}\right) K_{v}\left(\frac{2 \sqrt{z^{*}}}{\theta_{1}+2}\right)\right)^{-1} .
\end{aligned}
$$

For $\bar{P}_{1 D}\left(R_{D}, z\right)=0$, the similar kernel function is

$$
\begin{aligned}
& \Phi\left(r_{D}, z\right) \\
& =r_{D}^{\left(1-\beta_{0}\right) / 2} \\
& \quad \times\left(\left(\Psi_{v \cdot v}\left(r_{D}^{\left(\theta_{1} / 2\right)+1}, R_{D}^{\left(\theta_{1} / 2\right)+1}, \frac{2 \sqrt{z^{*}}}{\theta_{1}+2}\right)\right)\right. \\
& \quad \times\left(\left(1-\beta_{0}\right) \Psi_{v \cdot v}\left(1, R_{D}^{\left(\theta_{1} / 2\right)+1}, \frac{2 \sqrt{z^{*}}}{\theta_{1}+2}\right)\right. \\
& \left.\left.\quad-\sqrt{z^{*}} \Psi_{v+1 \cdot v}\left(1, R_{D}^{\left(\theta_{1} / 2\right)+1}, \frac{2 \sqrt{z^{*}}}{\theta_{1}+2}\right)\right)^{-1}\right) .
\end{aligned}
$$


For $d \bar{P}_{1 D} /\left.d r_{D}\right|_{r_{D} \rightarrow R_{D}}=0$, the similar kernel function is

$$
\begin{aligned}
\Phi\left(r_{D}, z\right) \\
=r_{D}^{\left(1-\beta_{0}\right) / 2} \\
\quad \times\left(\left(\frac{\left(1-\beta_{0}\right) \Psi_{v \cdot v}\left(r_{D}^{\left(\theta_{1} / 2\right)+1}, R_{D}^{\left(\theta_{1} / 2\right)+1}, 2 \sqrt{z^{*}} /\left(\theta_{1}+2\right)\right)+\sqrt{z^{*}} \Psi_{v \cdot v+1}\left(r_{D}^{\left(\theta_{1} / 2\right)+1}, R_{D}^{\left(\theta_{1} / 2\right)+1}, 2 \sqrt{z^{*}} /\left(\theta_{1}+2\right)\right)}{\left(1-\beta_{0}\right) \Psi_{v \cdot v}\left(1, R_{D}^{\left(\theta_{1} / 2\right)+1}, 2 \sqrt{z^{*}} /\left(\theta_{1}+2\right)\right)+\sqrt{z^{*}} \Psi_{v \cdot v+1}\left(1, R_{D}^{\left(\theta_{1} / 2\right)+1}, 2 \sqrt{z^{*}} /\left(\theta_{1}+2\right)\right)}\right)\right. \\
\quad \times\left(\left(1-\beta_{0}\right)-\frac{\sqrt{z^{*}}\left(1-\beta_{0}\right) \Psi_{v+1 \cdot v}\left(1, R_{D}^{\left(\theta_{1} / 2\right)+1}, 2 \sqrt{z^{*}} /\left(\theta_{1}+2\right)\right)+R_{D}^{\left(\theta_{1} / 2\right)+1} \Psi_{v+1 \cdot v+1}\left(1, R_{D}^{\left(\theta_{1} / 2\right)+1}, 2 \sqrt{z^{*}} /\left(\theta_{1}+2\right)\right)}{\left(1-\beta_{0}\right) \Psi_{v \cdot v}\left(1, R_{D}^{\left(\theta_{1} / 2\right)+1}, 2 \sqrt{z^{*}} /\left(\theta_{1}+2\right)\right)+\sqrt{z^{*}} R_{D}^{\left(\theta_{1} / 2\right)+1} \Psi_{v \cdot v+1}\left(1, R_{D}^{\left(\theta_{1} / 2\right)+1}, 2 \sqrt{z^{*}} /\left(\theta_{1}+2\right)\right)}\right) .
\end{aligned}
$$

From formation pressure equation (24), the solution's expression of wellbore pressure can be unified as follows:

$$
\bar{P}_{w D}\left(r_{D}, z\right)=\bar{q}_{D}(z) \frac{1}{z+(1 / \Phi(1, z))} .
$$

Substituting (25), (26), and (27) into (24) and (A.3), respectively, we can get similar structure of formation pressure and the wellbore pressure of the fractal dual-porosity reservoir with spherical flow under three types of outer boundaries (infinite, constant pressure, and closed) in the Laplace.

\section{The Similar Structure Method Algorithm (SSMA)}

This section presents the work of fluid mechanics in porous medium to establish reservoir seepage mathematical models according to the given formation, testing well, and fluid physical parameters. The formation pressure changing law with time of test well is obtained by solving these models. The well test analysis is on quantitative interpretation of the measurable pressure change combined with production data. Based on this test, we can obtain the parameters which cannot be directly measured. The parameters are important to calculate reservoir reserves and make scheme for the oil/gas field exploration, including pollution situation, improvement degree, wellbore damage, wellbore effect, flow coefficient and permeability of the test formation, and reservoir boundary.

For improvement of the modern well test analysis method, this paper programmed SSMA via SSM and Stehfest numerical inversion to calculate wellbore pressure and its derivative of reservoir seepage models. The algorithm is shown in Figure 1.

\section{The Pressure Transient Characteristics and Flow Regimes}

In this section, the dimensionless formation pressure and derivative responses for fractal dual-porosity reservoir spherical flow are calculated by SSMA proposed above.
The well test analysis cures of wellbore pressure and pressure derivative are drew out with $T_{D} / C_{D}$ as abscissa and $P_{w D}$, $P_{w D}^{\prime} \cdot T_{D} / C_{D}$ as ordinate. We analyze the effects of relevant parameters on the behavior.

Figure 2 shows the dimensionless formation pressure and derivative responses for fractal dual-porosity reservoir affected by fractal dimension $d_{f}$ in three types of outer boundaries. In early time wellbore storage period (stage 1) is observed in both pressure change and derivative aligned in unite slope trend which follow by a hump representing the transition flow (stage 2) to radial flow period (stage 3) impacted by the fractal dimension. After the radial flow, the fissure produces independently with the flow. This flow regime is followed by a characteristic dip in the pressure derivative, which is caused by cross flow (stage 4) in matrix. Finally, we attain the last-time compound radial flow (stage 5) characterized by different outer boundaries condition.

It should be noted that all the flow regimes described in the previous paragraph exist for all dual-porosity reservoirs. Depending on system properties, particularly wellbore storage parameter, some of the flow regimes above may be changed. In this section we present some results to reflect the effects of reservoir.

Figure 3 presents the dimensionless variables of wellbore storage and skin factor $C_{D} e^{2 s}$ on fractal dual-porosity reservoir with spherical flow. It can be seen that with all other parameters kept constant, the curve parameters $C_{D} e^{2 s}$ has obviously impacted on period of radial flow and cross flow. As the value of $C_{D} e^{2 s}$ increases, the duration time of radial flow in fissure decreases. When the faster cross flow takes place for the matrix to fissure, the earlier reflection on dimensionless curve appears.

Figure 4 shows the effect of fractal dimension $d_{f}$ on fractal dual-porosity reservoir with spherical flow. The properties of the reservoir are identical in this case. As shown in Figure 4 increasing the number of fractal dimension significantly influences the magnitude of the whole regime pressure and derivatives. That is because of the fact that increasing fractal dimension will improve the permeability around wellbore.

Figure 5 shows the effect of fractal exponential $\theta$ on fractal dual-porosity reservoir with spherical flow. As show in Figure 4, increasing the value of fractal exponent significantly 


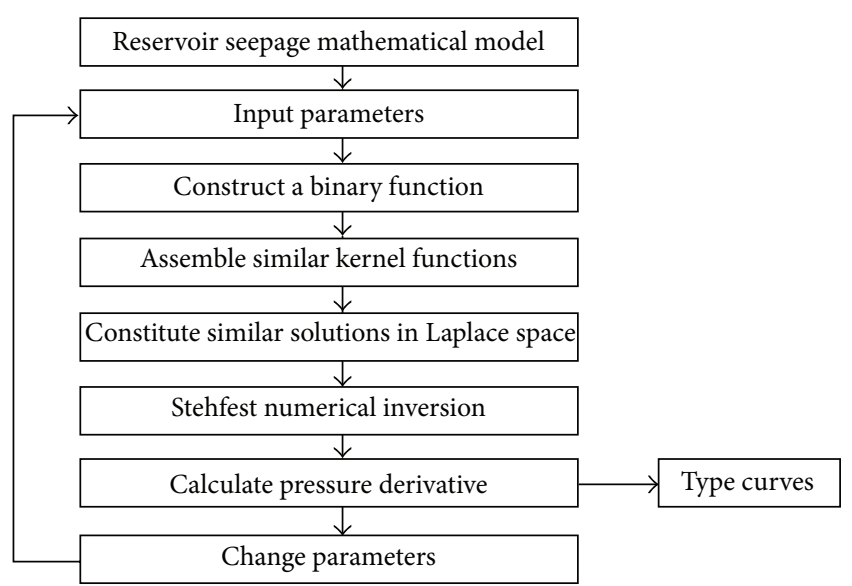

FIgURE 1: The similar structure method algorithm for solving the reservoir seepage models.

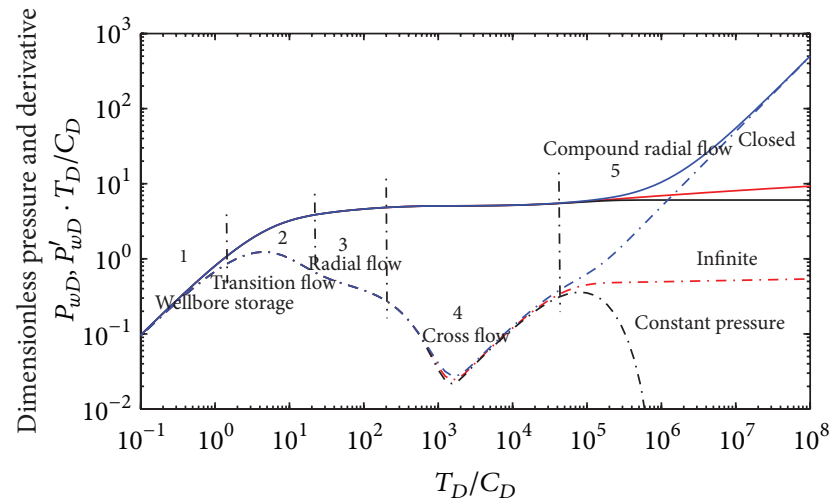

FIGURE 2: Well testing curves in fractal dual-porosity reservoir with three types of outer boundaries $(\omega=0.01, \lambda=0.00001, \theta=0.5$, $d_{f}=1.3, C_{D} e^{2 s}=3$, and $\left.R=500 \mathrm{~m}\right)$.

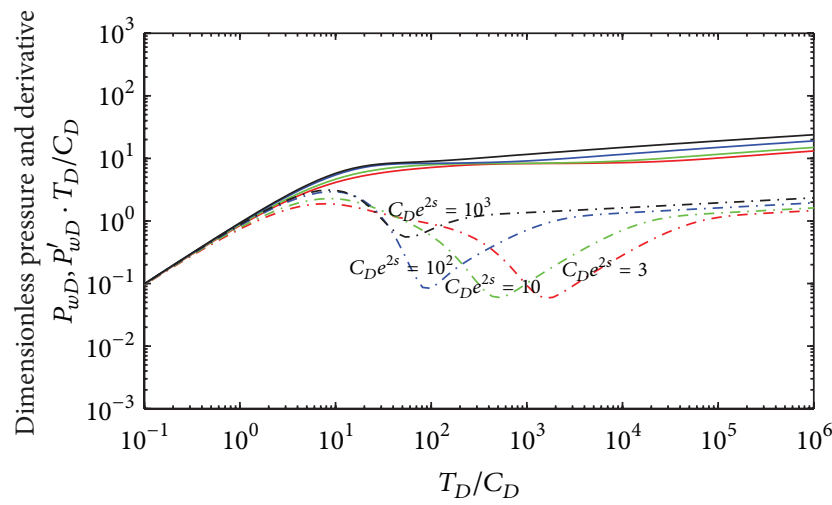

FIGURE 3: Effects of the curve parameters on pressure responses of a test well in fractal dual-porosity reservoir $(\omega=0.01, \lambda=0.00001$, $\theta=0.5, d_{f}=1.3$, and $R=500 \mathrm{~m}$ ).

influences the magnitudes of whole regime pressures and derivatives. Fractal exponent depicts the connectivity of fractal object. With the fractal exponent increasing, the pressure curve is on the upward trend. It shows that the more tortuous fissure system is, the worse connectivity is.

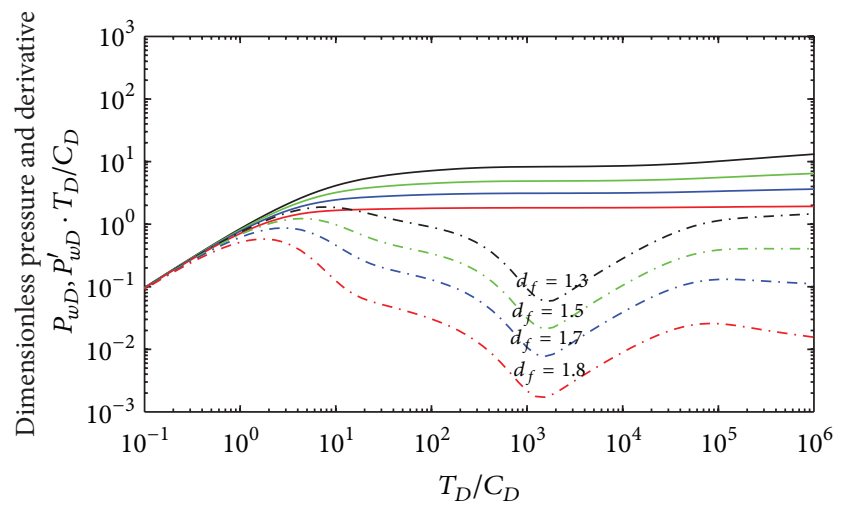

FIGURE 4: Effect of fractal demission on pressure responses of a test well in fractal dual-porosity reservoir $(\omega=0.01, \lambda=0.00001, \theta=$ $0.5, C_{D} e^{2 s}=3$, and $R=500 \mathrm{~m}$ ).

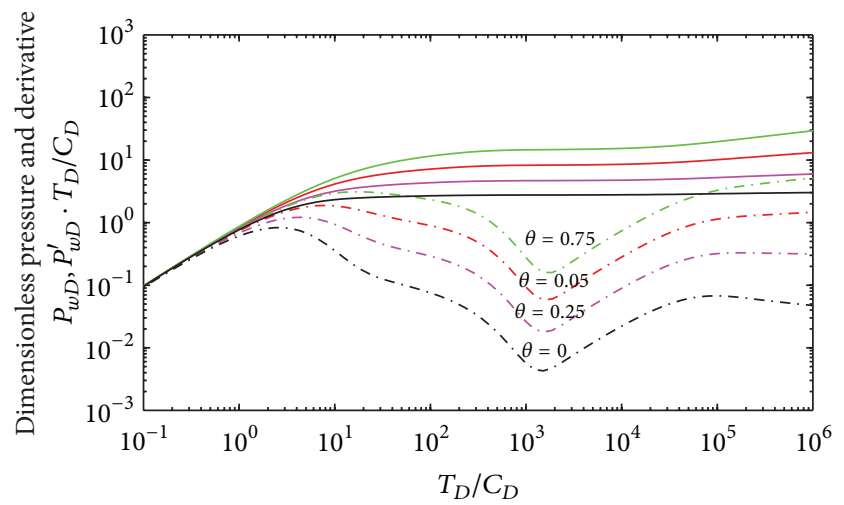

FIGURE 5: Effect of fractal exponent on the pressure responses of a test well in fractal dual-porosity reservoir $(\omega=0.01, \lambda=0.00001$, $d_{f}=1.3, C_{D} e^{2 s}=3$, and $R=500 \mathrm{~m}$ ).

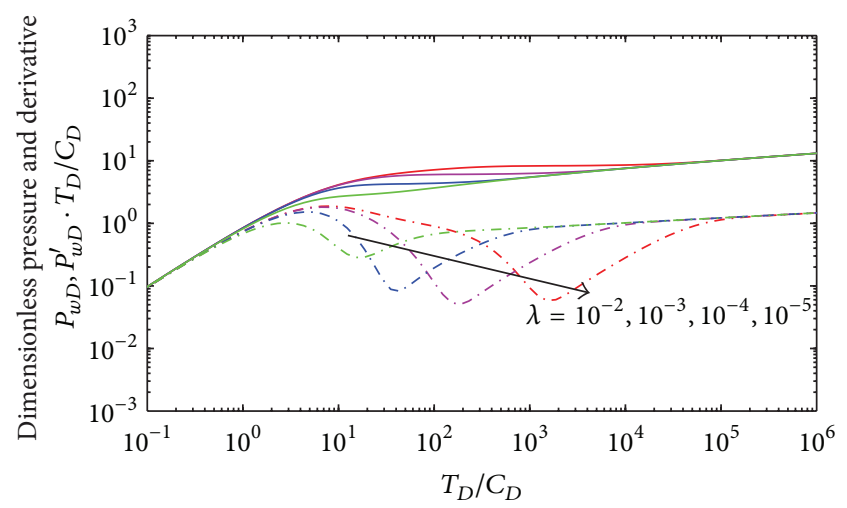

FIGURE 6: Effect of interporosity flow coefficient on the pressure responses of a test well in fractal dual-porosity $(\omega=0.01, \theta=0.5$, $d_{f}=1.3, C_{D} e^{2 s}=3$, and $\left.R=500 \mathrm{~m}\right)$.

Figure 6 shows the effect of interporosity $\lambda$ on fractal dual-porosity with spherical flow. Figure 6 indicates that the reflection of cross flow develops at later times for larger value of $\lambda$. Also, duration of the radial flow in fissure depends on the value of $\lambda$. This is understood as $\lambda$ is directly proportional to cross flow time of oil (gas) in matrix block. Faster 


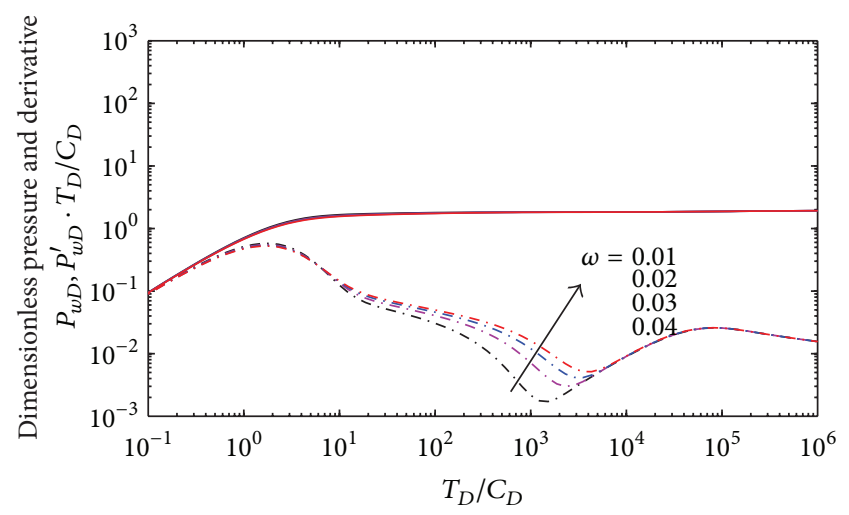

FIGURE 7: Effect of storativity ratio on the pressure responses of a test well in fractal dual-porosity reservoir $(\lambda=0.00001, \theta=0.5$, $d_{f}=1.3, C_{D} e^{2 s}=3$, and $\left.R=500 \mathrm{~m}\right)$.

of the cross flow takes palace, the reflection on the dimension curve appears earlier.

Figure 7 shows the effect of storativity ratio $\omega$ on fractal dual-porosity with spherical flow. The storativity significantly affects on the radial flow and period of cross flow. The smaller the storativity is, the deeper and wider the dip in the derivative becomes. The fissure system flowing (stage 2) sustains short time, which will be probability covered by period of the wellbore storage.

\section{Conclusion}

The SSM completed the theory of solutions' similar structure. When we deal with the boundary value problem, a general second-order linear differential equation, the formation of the similar structure is decided by the coefficients of the left (inner) boundary condition. The kern functions are achieved by the coefficients of the right (outer) boundary condition. So the SSM is proposed to solve such complicated problem in reality.

Many existing reservoir seepage fluid models can be adapted to the boundary value problem of the extended modified Bessel equation. In this paper, we propose SSM which is a convenient, effective, creative method to solve the problem of fluid flow in porous media since it can avoid complex procedures. The proposed SMMA optimizes the programming process of well test analysis software and also simplifies the processing of Laplace inversion.

When $d_{f_{1}}=d_{f_{2}}$ and $\theta=0(v=0)$, it is easy to get a dual-porosity reservoir mathematic model. With the solutions' similar structure on the model of fractal dual-porosity reservoir, it is easy to observe how the wellbore storage, skin factor, and fractal dimension affect the dimensionless formation pressure and the wellbore pressure. The model as well as the corresponding type curves are quite general that they are useful in predicting the production performance or analyzing the production data from this type of well-reservoir systems.

\section{Appendices}

\section{A. Derivation of Two Linearly Independent Solutions of the General Extended Modified Bessel Equation}

In this section, we demonstrate why (1) has two linearly independent solutions

$$
I_{v}\left(k x^{\beta}\right), \quad K_{v}\left(k x^{\beta}\right) .
$$

Substituting the bellow variables into (1)

$$
u=x^{-\alpha} y, \quad h=k x^{\beta},
$$

where $\alpha=(1-A) / 2, \beta=q / 2$, and $k=2 \sqrt{C} / q$, we can obtain the following standard Bessel equation:

$$
h^{2} \frac{d^{2} u}{d h^{2}}+h \frac{d u}{d h}-\left(h^{2}+v^{2}\right) u=0,
$$

where $v=\sqrt{(1-A)^{2}-4 B} / q$.

The general solution of (1) is

$$
u=C_{1} I_{v}(h)+C_{2} K_{v}(h),
$$

where $C_{1}$ and $C_{2}$ are arbitrary constants. Substituting (A.3) into (A.5), the general solution of (1) is

$$
y=x^{\alpha}\left[C_{1} I_{v}(h)+C_{2} K_{v}(h)\right] .
$$

We know that $I_{v}\left(k x^{\beta}\right), K_{v}\left(k x^{\beta}\right)$ are two linearly independent solutions for the extended modified Bessel equation (1).

\section{B. Mathematical Model of Fractal Dual-Porosity Reservoir}

The fissure media and matrix block in dual-porosity media are homogeneously distributed. Due to taking small size in reservoir volume, the fissure media has low porosity with high permeability. Whereas, the porosity of the matrix block is high with low permeability because of sedimentation and diagenesis. We can ignore the permeability of matrix block for simplified model.

To formulate the derivation of the mathematical mode for fractal dual-porosity reservoir with spherical flow, the basic assumptions used for fluid flow in porous media are as follows:

(i) flat pitch dual-porosity reservoir, uniform thickness, and isotropic;

(ii) single-phase micro compressible fluid, spherical flow which has one production or injection well;

(iii) darcy's law applies and isothermal curve;

(iv) neglecting the capillary single-phase horizontal flow without gravity effect;

(v) fracture network becomes deformed under the pressure with fractal feature and the variety of fractal dimension can be ignored. 
The permeability and porosity distributions of a fractal reservoir have been defined in various forms in different studies. Here, we consider skin factor's influence by $r_{w e}=$ $r_{w} e^{-s}$ and embed the fractal dimension $d_{f}$ of fractal network into the Euclid space with the dimension $d=2$. We make use of definitions similar to those proposed by [7]:

$$
\begin{gathered}
\phi_{i}=\phi_{w i}\left(\frac{r}{r_{w e}}\right)^{d_{f_{i}}-d} \quad(i=1,2), \\
k_{i}=k_{w i}\left(\frac{r}{r_{w e}}\right)^{d_{f_{i}}-\theta_{i}-d} \quad(i=1,2) .
\end{gathered}
$$

Darcy's law says that

$$
v_{i}=-\frac{k_{i}}{\mu} \Delta P_{i} \quad(i=1,2) .
$$

Due to the conservation law, the continuity equation of the flow in fissure media is described by the following partial differential equation:

$$
\frac{\partial\left(\phi_{1} \rho\right)}{\partial t}+\nabla\left(\rho v_{1}\right)-q_{\lambda}=0
$$

The flow in matrix block is described by the following partial differential equation:

$$
\frac{\partial\left(\phi_{2} \rho\right)}{\partial t}+q_{\lambda}=0 .
$$

In (B.5) and (B.6), the second item in the right-hand side represents the cross flow intensity between fissure media and matrix block and is defined as follows:

$$
q_{\lambda}=\frac{\alpha k_{2}}{\mu}\left(p_{2}-p_{1}\right) .
$$

Taking (B.2)-(B.4) and (B.7) into (B.5) yields

$$
\begin{aligned}
\frac{\partial^{2} p_{1}}{\partial r^{2}} & +\frac{\beta_{0}}{r} \frac{\partial p_{1}}{\partial r}+\alpha \frac{k_{w 2}}{k_{w 1}}\left(\frac{r}{r_{w e}}\right)^{\left(d_{f_{2}}-d_{f_{1}}\right)-\left(\theta_{2}-\theta_{1}\right)}\left(p_{2}-p_{1}\right) \\
& =\frac{\mu \phi_{w 1} C_{t_{1}}}{k_{w 1}}\left(\frac{r}{r_{w e}}\right)^{\theta_{1}} \frac{\partial p_{1}}{\partial t} .
\end{aligned}
$$

Taking (B.2)-(B.4) and (B.7) into (B.6) yields

$$
\frac{\phi_{w 1} C_{t_{2}}}{k_{w 1}}\left(\frac{r}{r_{w e}}\right)^{\theta_{2}} \frac{\partial p_{2}}{\partial t}+\frac{\alpha k_{w 2}}{\mu}\left(p_{2}-p_{1}\right)=0,
$$

where $\beta_{0}=d_{f_{1}}-\theta_{1}-d+2, C_{t_{i}}=C_{0}+C_{i}(i=1,2)$ and

$$
C_{0}=\frac{1}{\rho} \frac{\partial \rho}{\partial p_{2}}, \quad C_{i}=\frac{1}{\phi_{i}} \frac{\partial \phi_{i}}{\partial p_{i}} .
$$

Initial condition is as follows:

$$
p_{1}(r, 0)=p_{2}(r, 0)=p_{0}
$$

Inner boundary condition is as follows:

$$
\begin{aligned}
p_{w} & =p_{1}\left(r_{w e}, t\right), \\
\left.\left(r^{2} \frac{\partial p_{1}}{\partial r}\right)\right|_{r=r_{w e}} & =\frac{\mu}{\delta \pi k_{w 1}}\left[q(t)+C \frac{d p_{w}}{d t}\right] .
\end{aligned}
$$

Three types of outer boundary condition are as follows.

Case 1. Infinite pressure outer boundary condition is

$$
p_{1}(\infty, t)=p_{0}
$$

Case 2. Constant pressure outer boundary condition is

$$
p_{1}(R, t)=p_{0} .
$$

Case 3. Closed outer boundary condition is

$$
\left.\frac{\partial p_{1}}{\partial r}\right|_{r \rightarrow R}=p_{0} .
$$

In terms of convenience of calculation, we define the dimensionless variables as follows:

$$
\begin{aligned}
& P_{i D}=\frac{\delta \pi k_{w 1} r_{w e}}{3.685 \times 10^{-3} q_{e} \mu}\left(p_{0}-p_{i}\right) \quad(i=1,2), \\
& r_{D}=\frac{r}{r_{w e}}=\frac{r}{r_{w}} e^{s}, \quad R_{D}=\frac{R}{r_{w e}}=\frac{R}{r_{w}} e^{s}, \\
& q_{D}\left(t_{D}\right)=\frac{q(t)}{q_{e}}, \\
& t_{D}=\frac{3.6 k_{w 1} t}{\left(\phi_{w 1} C_{t_{1}}+\phi_{w 2} C_{t_{2}}\right) r_{w}^{2} \mu}, \\
& C_{D}=\frac{k_{w 1} e^{s}}{\delta \pi\left(\phi_{w 1} C_{t_{1}}+\phi_{w 2} C_{t_{2}}\right)}, \quad \lambda=\frac{\alpha r_{w}^{2} k_{w 2}}{k_{w 1}}, \\
& \omega=\frac{\phi_{w 1} C_{t_{1}}}{\phi_{w 1} C_{t_{1}}+\phi_{w 2} C_{t_{2}}}, \\
& T_{D}=\frac{t_{D}}{C_{D}}, \quad \beta_{i}=d_{f_{i}}-\theta_{i} \quad(i=1,2), \\
& \begin{cases}\delta=2 & \text { hemisphere, } \\
\delta=4 & \text { sphere. }\end{cases}
\end{aligned}
$$


All the parameters are explained in the nomenclature. The dimensionless mathematical model for fractal dual-porosity reservoir model can be obtained as follows:

$$
\begin{gathered}
\frac{\partial P_{1 D}}{\partial r_{D}^{2}}+\frac{\beta_{0}}{r_{D}} \frac{\partial P_{1 D}}{\partial r_{D}}+\frac{\lambda r_{D}^{\beta_{i}-\theta_{i}}}{e^{2 s}}\left(P_{2 D}-P_{1 D}\right)=\frac{\omega r_{\theta_{1}}}{C_{D} e^{2 s}} \frac{\partial P_{1 D}}{\partial T_{D}} \\
(1-\omega) r_{D}^{\theta_{1}} \frac{\partial P_{2 D}}{\partial T_{D}}+\lambda C_{D}\left(P_{2 D}-P_{1 D}\right)=0 \\
P_{1 D}\left(r_{D}, 0\right)=P_{2 D}\left(r_{D}, 0\right)=0 \\
\left.\left(r_{D D}^{2} \frac{\partial P_{w D}}{\partial r_{D}}\right)\right|_{r_{D}=1}=-P_{D} T_{D}+\frac{d P_{w D}}{d T_{D}} \\
\left.\left.P_{i D}\right|_{r_{D} \rightarrow \infty}=0, T_{D}\right), \\
\text { or } \quad P_{i D}\left(R_{D}, T_{D}\right)=0 \\
\text { or }\left.\quad \frac{\partial P_{i D}}{\partial R_{D}}\right|_{r \rightarrow R_{D}}=0 .
\end{gathered}
$$

Taking the Laplace transform of $P_{1 D}$ with respect to $T_{D}$, we get the boundary value problem of the ordinary differential equation with parameter $z$ (where $z$ denotes Laplace space variable); that is,

$$
\begin{gathered}
\frac{d^{2} \bar{P}_{1 D}}{d r_{D}^{2}}+\frac{\beta_{0}}{r_{D}} \frac{d \bar{P}_{1 D}}{d r_{D}}+\frac{\lambda r_{D}^{\beta_{i}-\theta_{i}}}{e^{2 s}}\left(\bar{P}_{2 D}-\bar{P}_{1 D}\right)=\frac{\omega r_{\theta_{1}}}{C_{D} e^{2 s}} \bar{P}_{1 D} \\
(1-\omega) r_{D}^{\theta_{1}} \bar{P}_{2 D}+\lambda C_{D}\left(\bar{P}_{2 D}-\bar{P}_{1 D}\right)=0 \\
\bar{P}_{1 D}\left(r_{D}, 0\right)=\bar{P}_{2 D}\left(r_{D}, 0\right)=0, \\
\bar{P}_{w D}=\bar{P}_{1 D}\left(1, T_{D}\right), \\
\left.\left(r_{D}^{2} \frac{d \bar{P}_{w D}}{d r_{D}}\right)\right|_{r_{D}=1}=-\bar{q}_{D}(z)+z \bar{P}_{w D}, \\
\left.\bar{P}_{i D}\right|_{r_{D} \rightarrow \infty}=0 \\
\text { or }\left.\bar{P}_{i D}\right|_{r_{D}=R_{D}}=0 \\
\text { or }\left.\frac{\left.d \bar{P}_{i D}\right|_{r \rightarrow R_{D}}=0 .}{d r_{D}}\right|_{r}=0
\end{gathered}
$$

\section{Nomenclature}

C: Wellbore storage coefficient, $\mathrm{m}^{3} / \mathrm{Pa}$

$C_{0}$ : Fluid compressibility, $\mathrm{Pa}^{-1}$

$C_{t_{i}}$ : Compressibility, $\mathrm{Pa}^{-1}$

$d$ : Euclid dimension

$d_{f_{i}}:$ Fractal dimension

$k_{i}$ : Permeability, $\mathrm{m}^{2}$ $k_{w i}: \quad$ Initial permeability, $\mathrm{m}^{2}$

$p_{i}: \quad$ Reservoir pressure, $\mathrm{Pa}$

$p_{w}: \quad$ Wellbore pressure, $\mathrm{Pa}$

$p_{0}$ : Initial reservoir pressure, $\mathrm{Pa}$

$r$ : Radial distance in spherical coordinate, $\mathrm{m}$

$R: \quad$ Radial distance of outer boundary radius, $\mathrm{m}$

$s$ : Skin factor, dimensionless

$t$ : Time, $\mathrm{m}$

$v_{i}: \quad$ Spherical flow velocity, $\mathrm{m} / \mathrm{s}$

$\rho: \quad$ Fluid density, $\mathrm{kg} / \mathrm{m}^{3}$

$\alpha$ : Form factor

$\theta_{i}: \quad$ Fractal exponent, dimensionless

$\mu$ : Viscosity of fluid, Pa.s

$\phi_{i}$ : Porosity, fraction

$\phi_{w i}:$ Initial porosity, fraction

$\omega: \quad$ Storativity ratio, dimensionless

$\lambda: \quad$ Interporosity flow coefficient, dimensionless

$I_{v}(\cdot)$ : Modified Bessel function of first-order $v$

$K_{v}(\cdot)$ : Modified Bessel function of second-order $v$

$\varphi(\cdot)$ : A binary function

$\Phi(\cdot)$ : Similar kernel function

$z$ : $\quad$ Laplace space variable.

\section{Superscript}

-: Laplace domain.

Subscript

$$
\begin{array}{ll}
i=1: & \text { Fissure media } \\
i=2: & \text { Matrix block } \\
D: & \text { Dimensionless } \\
o: & \text { Initial condition } \\
w: & \text { Wellbore parameter } \\
w e: & \text { Well effective. }
\end{array}
$$

\section{Conflict of Interests}

The authors declare that they do not have any commercial or associative interest that represents a conflict of interests in connection with the work submitted.

\section{Acknowledgments}

This study is supported by National Natural Science Foundation for Petroleum and Chemical Industry Unite Fund Support Key Projects (Grant no. U1262209), the National Natural Science Fund (Grant no. 51274172), and the Scientific Research Fund of Sichuan Education Provincial Department of China (Grant no. 12ZA164).

\section{References}

[1] L. Xu and S. C. Li, "Similar structure of flow effective well radius model through homogeneous reservoir solutions," Journal of Chongqing Normal University, vol. 28, pp. 32-34, 2011. 
[2] L.-H. Zhang, J.-J. Guo, and Q.-G. Liu, "A new well test model for a two-zone linear composite reservoir with varied thicknesses," Journal of Hydrodynamics, vol. 22, no. 6, pp. 804-809, 2010.

[3] B. B. Mandelbrot, The Fractal Geometry of Nature, W. H. Freeman and Co., New York, NY, USA, 1982.

[4] T.A. Hewett, "Fractal distribution of reservoir heterogeneity and their influence on fluid transport," in Proceedings of the SPE Annual Technical Conference and Exhibition (ATCE '86), Document ID 15386, p. 16, SPE, New Orleans, La, USA, October 1986.

[5] J. Chang and Y. C. Yortsos, "Pressure transient analysis of fractal reservoirs," in Proceedings of the SPE Annual Technical Conference and Exhibition (ATCE '88), Document ID 18170, SPE, Houston, Tex, USA, October 1988.

[6] J. Chang and Y. C. Yortsos, "Pressure transient analysis of fractal reservoirs," SPE Formation Evaluation, vol. 5, no. 1, pp. 31-38, 1990.

[7] R. A. Beier, "Pressure transient model of a vertically fractured well in a fractal reservoir," in Proceedings of the 65th SPE Annual Technical Conference and Exhibition (ATCE '90), Document ID 20582, pp. 557-566, New Orleans, La, USA, September 1990.

[8] S. Aprilian, D. Abdassah, L. Mucharam, R. Sumatri, and R. D. Pertamina, "Application of fractal reservoir model for interference test analysis in Kamojang geothermal field (Indonesia)," in Proceedings of the SPE Annual Technical Conference and Exhibition (ATCE '93), Document ID 26465, p. 10, Houston, Tex, USA, October 1993.

[9] D.-K. Tong and R.-H. Wang, "The line-source solution and flow analysis of fluid in fractal reservoir," Journal of Hydrodynamics, vol. 10, no. 4, pp. 59-65, 2002.

[10] Y.-G. Zhang and D.-K. Tong, "The pressure transient analysis of deformation of fractal medium," Journal of Hydrodynamics, vol. 20, no. 3, pp. 306-313, 2008.

[11] G. I. Barenblatt, Y. P. Zheltov, and I. N. Kochina, "Basic concepts on the theory of seepage of homogeneous liquids in fissured rock," Prikladnaya MatematIka I MekhanIka, vol. 24, no. 5, pp. 852-864, 1960.

[12] W. A. Abbott, T. Collins, D. B. Tipple, H. K. van Poollen, and Associates Inc, "Practical application of spherical low transient analysis," in Proceedings of the SPE Annual Fall Technical Conference and Exhibition, 7485-MS, p. 11, Houston, Tex, USA, October 1987.

[13] P. S. Zheng, S. C. Li, and Y. F. Zhang, "The solutions structure of a type of ordinary differential equation system with closed right boundary conditions," Journal of Xihua University, vol. 24, no. 6, pp. 88-94, 2005.

[14] J. C. Wang, S. C. Li, and L. Xu, "Spherical fluids flow models in composite reservoir based on similar structure of solution," Journal of Guilin University of Technology, vol. 32, no. 4, pp. 624627, 2011.

[15] W. Li, X. P. Li, and S. C. Li, "Similar structure of the solution of mathematical model for the nonlinear flow of fractal commingled oil reservoirs," Petroleum Geology Oilfield Development in Daqing, vol. 31, no. 6, pp. 79-83, 2012.

[16] H. Stehfest, "Numerical inversion of laplace transforms," Communications of the ACM, vol. 13, no. 1, pp. 47-49, 1970. 


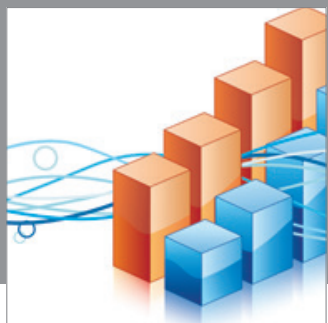

Advances in

Operations Research

mansans

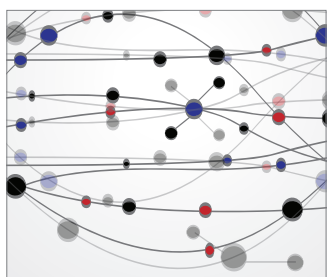

The Scientific World Journal
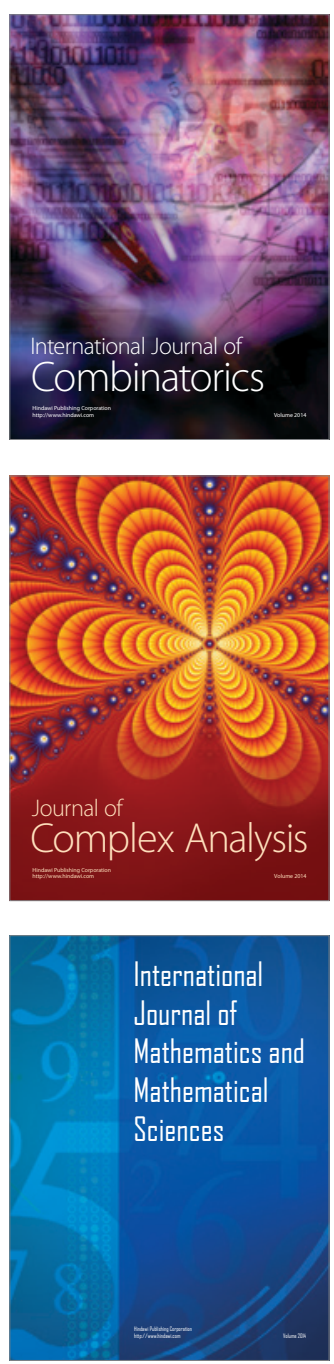
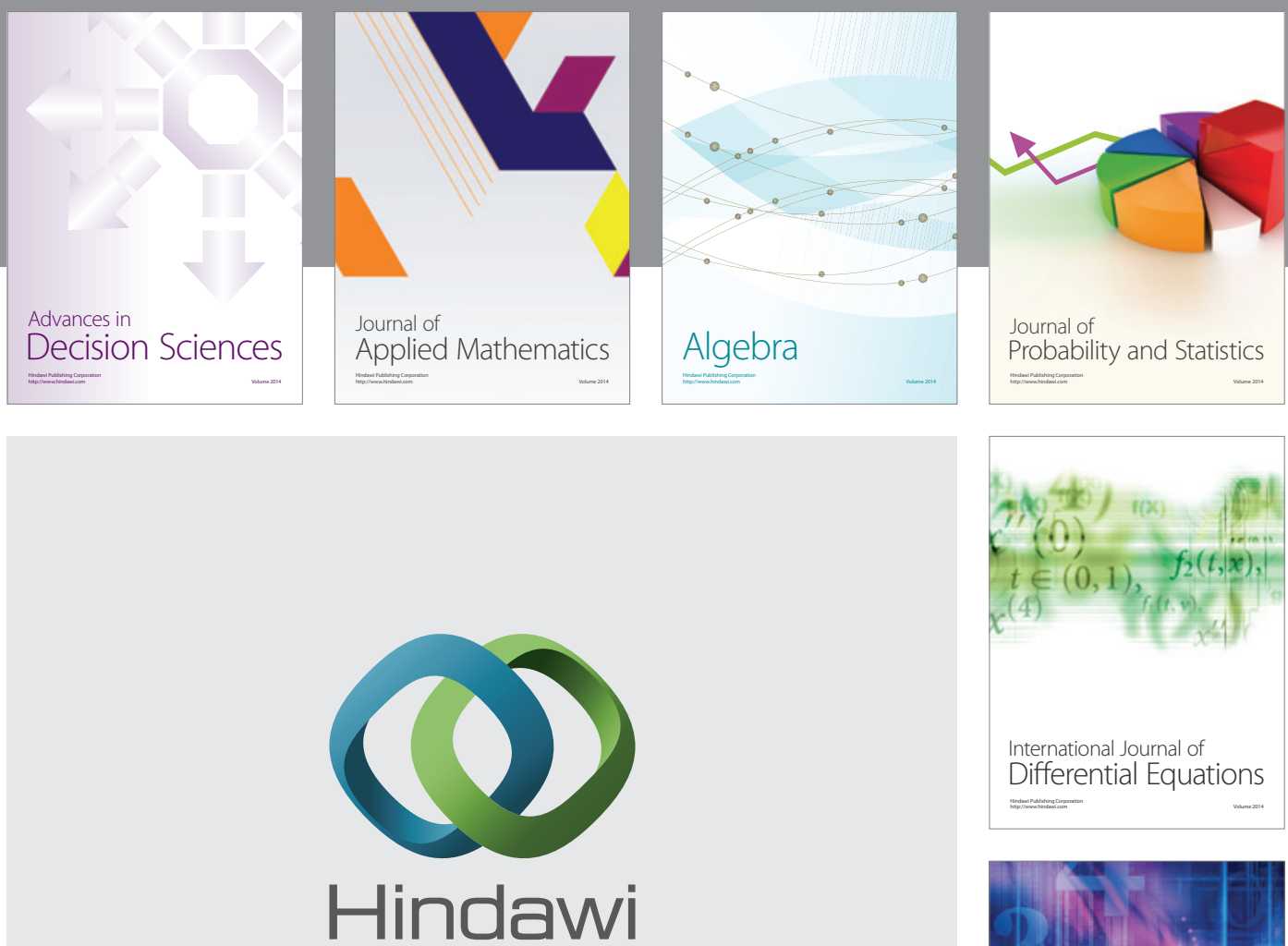

Submit your manuscripts at http://www.hindawi.com
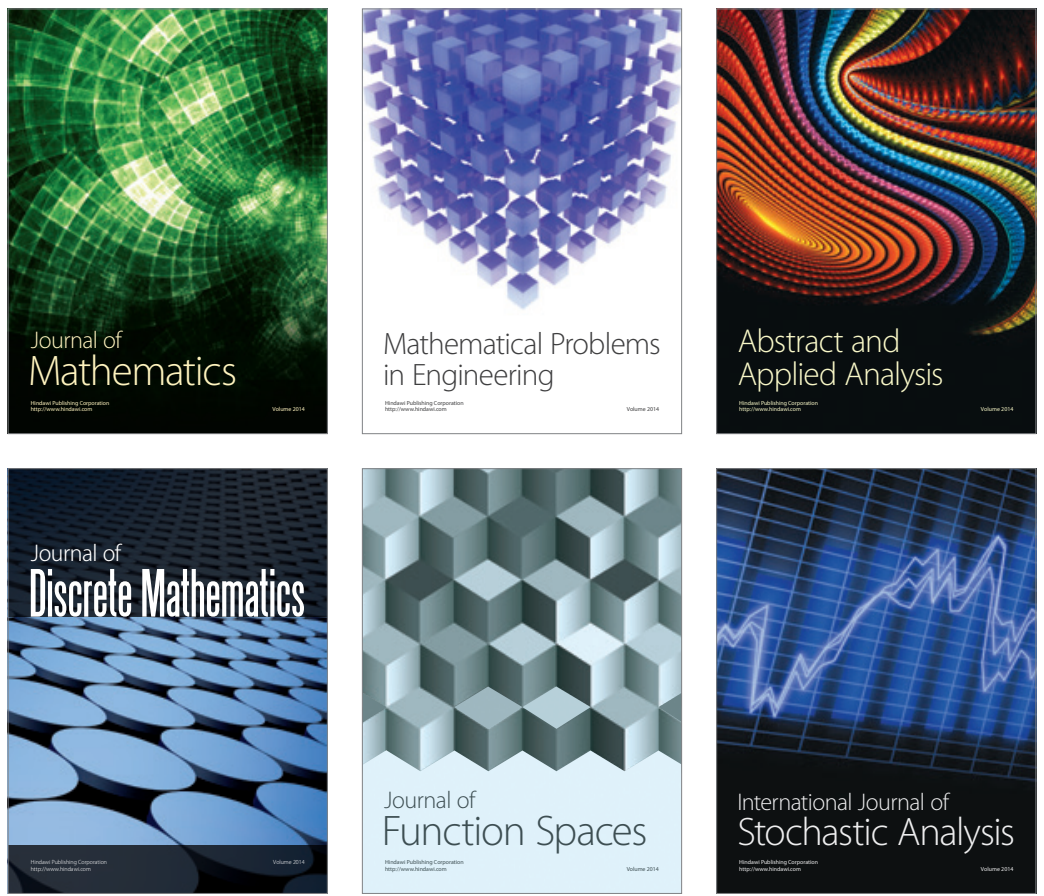

Journal of

Function Spaces

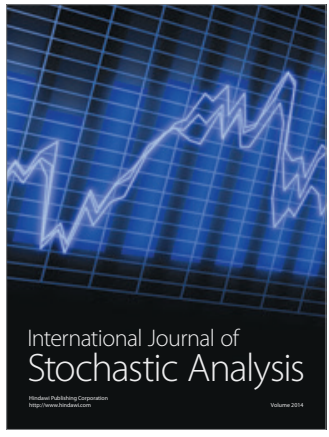

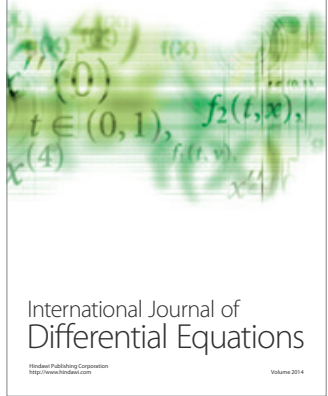
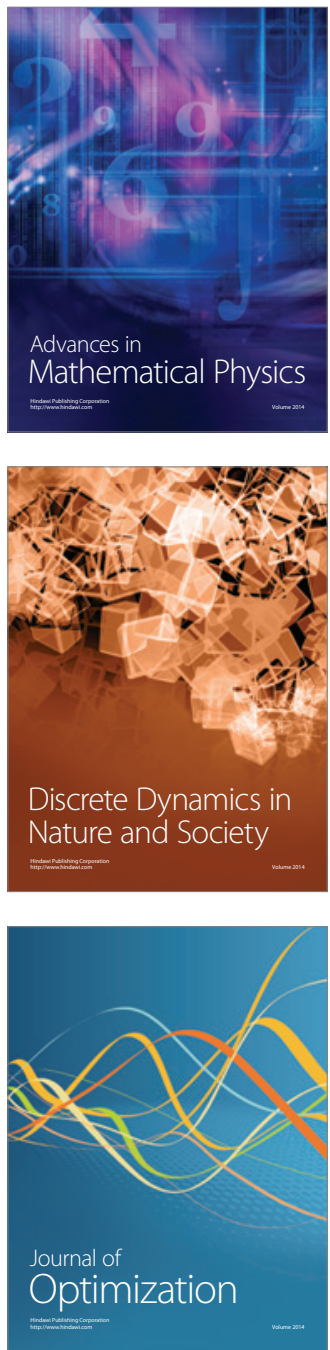